\title{
Supportability for Beyond Low Earth Orbit Missions
}

\author{
William Cirillo ${ }^{1}$ and Kandyce Goodliff ${ }^{2}$ \\ NASA Langley Research Center, Hampton, VA, 23681 \\ Gordon Aaseng ${ }^{3}$ \\ NASA Ames Research Center, Moffett Field, CA, 94035 \\ Chel Stromgren ${ }^{4}$ \\ Binera, Inc., Silver Springs, MD, 20910 \\ and \\ Andrew Maxwell ${ }^{5}$ \\ Georgia Institute of Technology, Hampton, VA 23666
}

\begin{abstract}
Exploration beyond Low Earth Orbit (LEO) presents many unique challenges that will require changes from current Supportability approaches. Currently, the International Space Station (ISS) is supported and maintained through a series of preplanned resupply flights, on which spare parts, including some large, heavy Orbital Replacement Units (ORUs), are delivered to the ISS. The Space Shuttle system provided for a robust capability to return failed components to Earth for detailed examination and potential repair. Additionally, as components fail and spares are not already on-orbit, there is flexibility in the transportation system to deliver those required replacement parts to ISS on a near term basis. A similar concept of operation will not be feasible for beyond LEO exploration. The mass and volume constraints of the transportation system and long envisioned mission durations could make it difficult to manifest necessary spares. The supply of on-demand spare parts for missions beyond LEO will be very limited or even non-existent. In addition, the remote nature of the mission, the design of the spacecraft, and the limitations on crew capabilities will all make it more difficult to maintain the spacecraft. Alternate concepts of operation must be explored in which required spare parts, materials, and tools are made available to make repairs; the locations of the failures are accessible; and the information needed to conduct repairs is available to the crew. In this paper, ISS heritage information is presented along with a summary of the challenges of beyond LEO missions. A number of Supportability issues are discussed in relation to human exploration beyond LEO. In addition, the impacts of various Supportability strategies will be discussed. Any measure that can be incorporated to reduce risk and improve mission success should be evaluated to understand the advantages and disadvantages of
\end{abstract}

\footnotetext{
${ }^{1}$ Senior Researcher, Space Missions Analysis Branch, MS 462, non-AIAA Member.

${ }^{2}$ Aerospace Engineer, Space Missions Analysis Branch, MS 462, AIAA Senior Member.

${ }^{3}$ Computer Systems Engineer, Intelligent Systems Division, non-AIAA Member.

${ }^{4}$ Vice President/Chief Scientist, 912 Thayer Avenue Suite 209, AIAA Member.

${ }^{5}$ Graduate Research Student, 100 Exploration Way, AIAA Student Member.
} 


\section{implementing those measures. Finally, an effort to model and evaluate Supportability for beyond LEO missions will be described.}

$\begin{array}{ll}\text { DSV } & \text { Nomenclature } \\ E B F^{3} & =\text { Electron Beam Freeform Fabrication } \\ E C L S S & =\text { Environmental Control and Life Support System } \\ E V A & =\text { Extra-Vehicular Activity } \\ I S S & =\text { International Space Station } \\ L E O & =\text { Low Earth Orbit } \\ L O C & =\text { Loss of Crew } \\ L O M & =\text { Loss of Mission } \\ M D M & =\text { Multiplexer-Demultiplexers } \\ N A S A & =\text { National Aeronautics and Space Administration } \\ O R U & =\text { Orbital Replacement Unit } \\ S M E & =\text { Subject Matter Expertise }\end{array}$

\section{Introduction}

$\mathrm{O}$ VER the past several years, the National Aeronautics and Space Administration (NASA), along with a number of its International Partners, has been working to establish a preliminary set of human and robotic space exploration architecture options for missions beyond the bounds of low Earth orbit (LEO). In addition to evaluating conceptual space transportation and surface system element options for a variety of exploration destinations, NASA has begun to develop a modeling capability to help establish a viable and robust Integrated Logistics Support system. Integrated Logistics Support, or Supportability, objectives are "to ensure that the product system is supported during development and operations in a cost-effective manner." $\mathrm{i}$ For the purposes of this paper, Supportability encompasses reliability, maintainability, reparability, redundancy and sparing philosophy.

Because of the unique mission requirements associated with long-duration human spaceflight missions beyond LEO, it is fundamentally important to establish during this early conceptual design phase a preliminary understanding of Supportability expectations that will eventually manifest themselves during the future hardware development and operations phases. If done well, this early Supportability modeling will aid in reducing the uncertainty associated with establishing a preliminary understanding of both mission support requirements and mission concepts for addressing these requirements.

Traditionally, NASA's human spaceflight missions have either been of short duration, such as the Mercury, Gemini, and Apollo missions, or if of a long duration, such as the International Space Station (ISS), in a destination (LEO) that was readily supportable by a number of redundant transportation systems. Today though, the international space community's space exploration architecture options ${ }^{\text {ii }}$ are primarily focused on a set of options that combine both long mission durations with destinations that will not be easily supportable once the mission has departed from its staging point. Because of these factors, NASA has recognized the importance of establishing early in the design life-cycle a preliminary understanding of the long-term mission support needs that will increase the probability of satisfying the challenging set of goals and objectives associated with these future human spaceflight activities.

This paper describes the current ISS Supportability approach and Supportability challenges for beyond LEO destinations in Section 2. Section 3 discusses the major Supportability issues for beyond LEO missions and introduces key concepts in evaluating Supportability. Various strategies for improving Supportability of beyond LEO missions are outlined in Section 4. Section 5 provides a look at the model plan and forward work to complete the model.

\section{Background}

\section{A. International Space Station Approach}

Most new space systems designs draw from existing and heritage systems because of the reduced risk expected from components and subsystems with substantial flight history. As a result, it is instructive to look at the International Space Station (ISS) to assess its approach to Supportability as it represents the current system for 
sustaining long-term human exploration beyond Earth. The strategy for continued safe operation for the ISS revolves around preventative and corrective maintenance. This approach is supported by a logistics train that allows necessary components for maintenance and repair to be brought from Earth. By relying on resupply from Earth, the ISS does not need to keep all spares on hand at all times but can time non-critical replacements with other scheduled missions. However, there are a large number of critical spares onboard ISS that are stored until required. For deep space missions, there might not be a differentiation between critical and non-critical spares as there will not be a means of resupply to transport the non-critical spares.

Scheduled or preventative maintenance is a major driver of Supportability related actions by astronauts onboard the ISS. Figure 1 shows the number of scheduled maintenance actions per month that were performed at the station through January 2011 (data provided by ISS Logistics and Maintenance Office). Failures and suspected failures requiring corrective action are the other main source of remove and replace tasks. Figure 2 shows the monthly count for maintenance actions related to failures and suspected failures. Although the deep space vehicle utilized for beyond LEO missions may be less complex than the ISS, analogs such as ISS are useful for understanding the importance of designing these missions with Supportability in the conceptual phase.

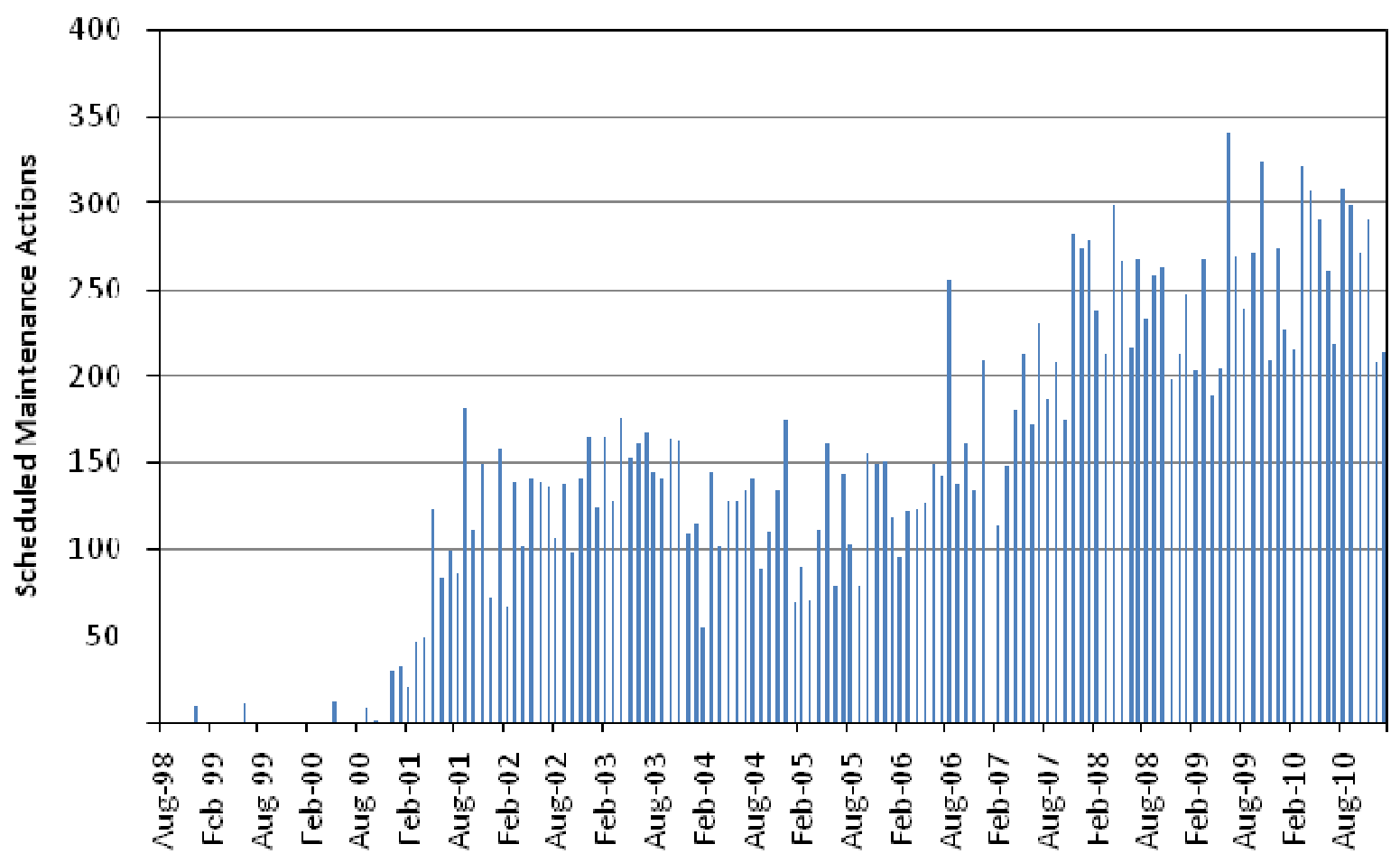

Figure 1 - Number of Scheduled Maintenance Actions per Month on the ISS 


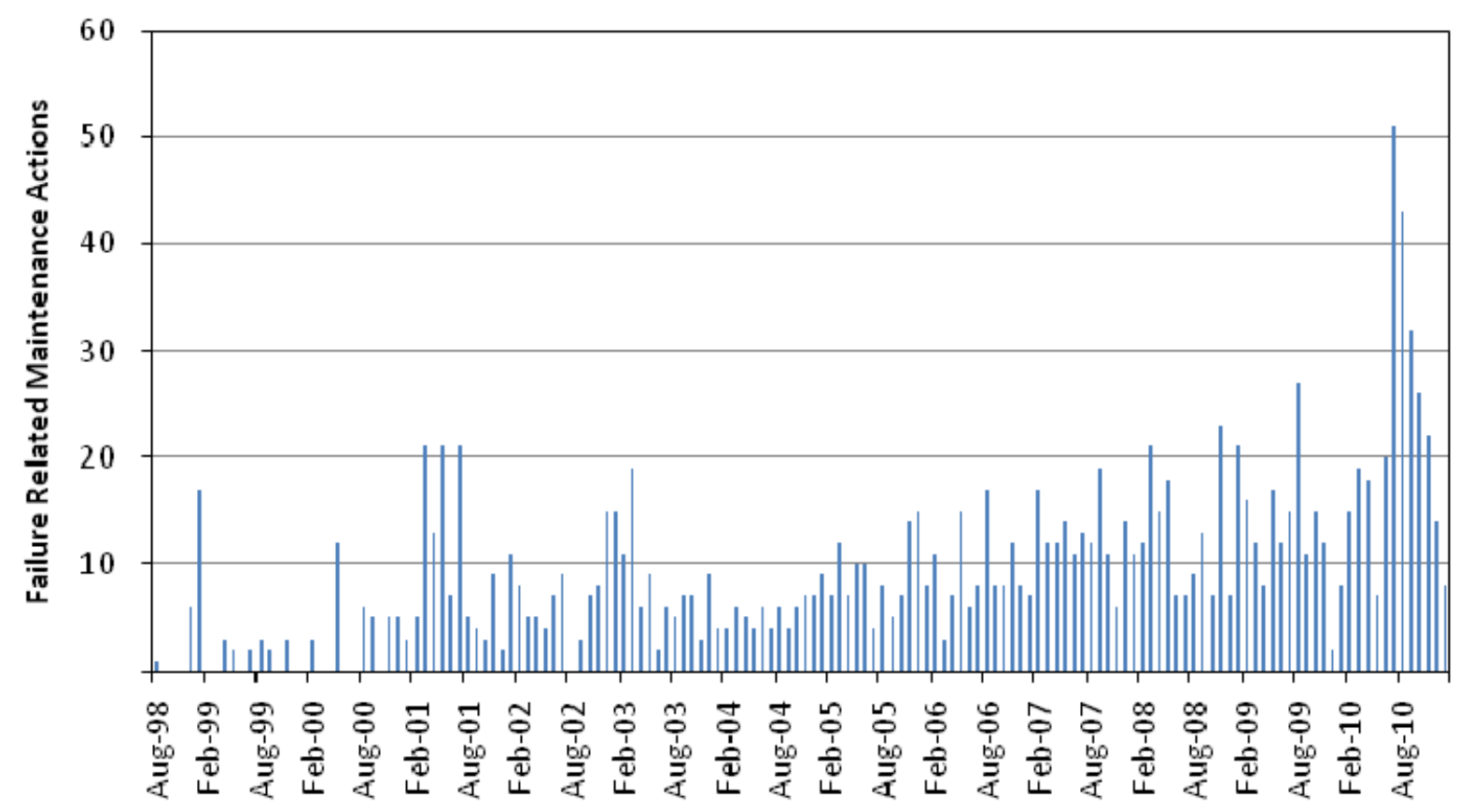

Figure 2 - Number of Maintenance Actions Related to Failures and Suspected Failures per Month on the ISS

\section{B. Beyond Low Earth Orbit Missions}

Currently, NASA is analyzing the requirements for beyond low Earth orbit (LEO) missions, including missions to near-Earth asteroids and the Mars system. The ISS approach for resupply will not work for these missions primarily due to the energy and time required to reach these destinations. Near-Earth asteroid missions and Mars system missions are on the order of months to years in duration from launch to landing back on Earth. Re-supply during these missions will likely not be feasible. Most missions will need to minimize cost by minimizing the number of launch vehicles required. This will reduce the amount of mass that can be delivered or even prepositioned for these missions. In addition, most missions will more than likely have severe volume limitations for logistics when considering all the elements to be sent to support the crew mission, such as habitation, power, life support, and science equipment. For these destinations, this will be the first time humans have traveled outside of the Earth's magnetosphere and will be exposed to a harsh radiation environment. This means it will be more difficult to estimate the failure rates of systems and components for a deep space vehicle. Finally, most missions beyond LEO will not include a quick abort path back to Earth. This dramatically increases the criticality of spacecraft systems and increases the demands on overall spacecraft reliability. All of these factors will have to be considered when designing a Supportability philosophy for missions beyond LEO in addition to destination specific challenges.

\section{Issues}

There will be a number of direct impacts of Supportability on deep space mission hardware and operations. The level of achieved Supportability will affect the probability of system failure and mission risk. Supportability requirements will drive spacecraft design and system accessibility. In addition, Supportability will directly impact time requirements on the crew to maintain and repair systems. Finally, Supportability will have a major impact on the amount of maintenance and spares that must be manifested on the mission in order to ensure the safety of the crew and the reliability of the mission.

When discussing Supportability for deep space missions, it is tempting to equate improved Supportability directly with increased reliability. The thinking is that if significant improvements in the reliability of all of the deep space vehicle (DSV) systems can be realized, then each of the areas described above will be improved. This view over-simplifies the factors that go into making the DSV supportable. While reliability is one contributor in safety, crew time, and spares requirements, there are other factors that will also control these areas.

Of the areas described above, one that is of particular concern in terms of mission design is the amount of spares that must be carried along with mission. Because there is little or no re-supply during these missions and because the deep space systems will be relatively complex, the expectation is a significant amount of spares will have to be 
flown along with the mission. The amount of required spares could be a major driver in the total mass and volume of the DSV.

While the amount of required spares is influenced by the reliability of systems, reductions in spares is relatively insensitive to increases in reliability. The total amount of spares that are required on-board the spacecraft is not driven by the actual amount of expected component failures. Given the reliability of the systems, the actual number of components that are expected to fail could be quite small. Rather, the amount of required spares is driven by the number of components that potentially could fail.

There are an enormous number of potential failures that can occur in a complex DSV. A significant number of those potential failures can endanger the mission and the crew. If any failure exceeds an established probability of occurrence, then that failure must be protected against. These are called "critical failures". For all critical failures, there must be a redundant capability, a work-around, or the ability to repair or replace the failed item.

It is the sheer number of possible failures that drives the amount of required spares. While any particular failure has a low probability of occurrence, the total failure rate can be significant. Since it is impossible to predict exactly which failures will occur, it is necessary to protect against all critical failures. The result is that only a small fraction of the manifested spares will actually be used. As an example, on the ISS, it is estimated that over $95 \%$ of the spares that are manifested (not including planned maintenance items) will not be used.

On ISS, where there is the capability to supply spares within a fairly short time frame, only a fraction of the total critical spares need to be manifested on-board. Only the truly critical spares (those which must be replaced within a short period of time or which would prevent a re-supply ship from docking) must be manifested. For deep space missions, where likely no spares will be delivered during the mission, a significantly larger fraction of critical spares would have to be carried on board the spacecraft.

Increases in reliability, even substantial increases, may only reduce the amount of required spares to some limited degree. For a given failure, if the probability of occurrence still exceeds the established threshold, then the failure must still be protected against. Getting closer to, but still remaining above, the limit does not reduce the amount of required spares.

Certainly, increasing the reliability of systems will reduce the probability of some failures to the point where they are no longer considered to be critical. The spares to protect against those failures would no longer need to be manifested. However, because of the number of potential failures, the threshold for component reliability must be very small.

A quick numerical example can be used to illustrate this concern. This example is not meant to represent an actual spacecraft system, but rather illustrate the influence that the number of potential failures has on the overall reliability. If the DSV had 1,000 potential failure types, each with an annual failure rate of 1 in 100,000, then the total probability of system failure in a one-year mission would be nearly $1 \%$.

Component reliabilities for spacecraft systems, even using current technologies, are already very high. Improving them to a degree where there is virtually no chance of failure will be technically very challenging. In addition, significant increases in reliability may also require increases in the mass and volume of the components, negating any reduction in spares.

Ultimately, there are a number of aspects of Supportability that will impact DSV design, sparing, crew requirements, and risk. These include:

- Reliability - The ability of the components and systems to keep operating in good working order over time.

- Maintainability - The characteristics of the components and systems that allow them to be maintained in good working order during the mission, maintaining performance and preventing failures. Improved maintainability may be a function of maintenance operations, monitoring, and failure prediction.

- Reparability - The characteristics of components and systems that facilitate repair by the crew following failure. Aspects of reparability include accessibility, level of repair, tool and equipment requirements, and crew skill requirements. A major factor in reparability for spacecraft is whether repairs must be conducted as part of an extra-vehicular activity (EVA) or whether they can be accomplished inside the spacecraft.

- Redundancy - The provision of additional or duplicate systems, equipment, etc., that function in case an operating part or system fails.

- Sparing Philosophy - Manifesting decisions made prior to the mission about what type and quantity of spares are sent along on the mission. Sparing philosophy will include not only the spares to be manifested but also the level of sparing, i.e. whether spares are manifested at the component level, the board level, or at some other level of repair. 


\section{Strategies for Improving Supportability}

In-flight Supportability can potentially be improved by incorporating several strategies into the architecture and mission design. These strategies must be evaluated from early in concept formulation, elaborated in system requirements, included in the procurement strategy and analyzed for optimum impact throughout system design. The strategy elements that will be described are:

- Level of repair

- Commonality

- Assembly repair - Ability to repair and supply prior to destination departure

- Redundancy - Complete and component-level

- Reliability

- In-space manufacturing

- Cannibalization and asset reallocation (in-situ resource utilization).

A summary of the benefits and risks are presented in Table 1 and elaborated in the following sections.

Table 1 - Supportability Strategy Elements

\begin{tabular}{|c|c|c|}
\hline Maintainability Strategy & Benefits & Risks \\
\hline Lower Level of Repair & - $\quad$ Reduced spares mass/volume & $\begin{array}{l}\text { - More difficult repairs require } \\
\text { more skill and training } \\
\text { - } \quad \text { More tools and test equipment } \\
\text { Increased crew time } \\
\text { requirements }\end{array}$ \\
\hline Commonality & $\begin{array}{ll}\text { - } & \text { Reduced spares mass/volume } \\
\text { - } & \text { Reduced development cost }\end{array}$ & $\begin{array}{l}\text { Design may not be optimum for } \\
\text { some uses } \\
\text { Difficult to manage across } \\
\text { multiple prime contractors and } \\
\text { development schedules }\end{array}$ \\
\hline Repair During Assembly & $\begin{array}{l}\text { - Minimize spares launch mass } \\
\text { for spares used in Earth orbit } \\
\text { - Assures full complement of } \\
\text { spares for the post-assembly } \\
\text { phase }\end{array}$ & $\begin{array}{l}\text { Requires planning for } \\
\text { contingency mass on some } \\
\text { launches }\end{array}$ \\
\hline Redundancy & $\begin{array}{l}\text { Inter-module redundancy can } \\
\text { reduce spares mass and volume } \\
\text { - Functional redundancy between } \\
\text { modules can reduce criticality } \\
\text { of failures in individual modules } \\
\text { - Complements a commonality } \\
\text { strategy }\end{array}$ & 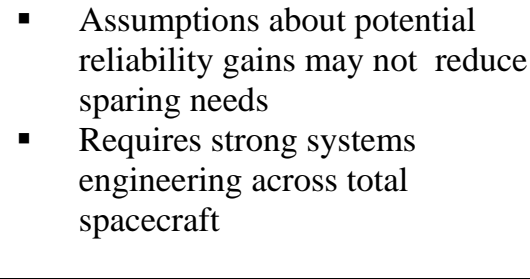 \\
\hline Reliability & $\begin{array}{l}\text { Reduces number of failures that } \\
\text { must be protected against } \\
\text { - } \\
\text { Reduces planned maintenance } \\
\text { actions and mass }\end{array}$ & $\begin{array}{l}\text { - Assumptions about potential } \\
\text { reliability gains may not reduce } \\
\text { sparing needs }\end{array}$ \\
\hline In-Space Manufacturing & $\begin{array}{l}\text { Mitigation of unexpected } \\
\text { failures } \\
\text { - } \quad \text { Possible reduced spares mass } \\
\text { and volume }\end{array}$ & $\begin{array}{l}\text { - } \quad \text { Tools are complex } \\
\text { Mass and power required are } \\
\text { considerable, and increase with } \\
\text { capability/flexibility } \\
\text { - Most likely failures are } \\
\text { probably not amenable to in- } \\
\text { space manufacturing }\end{array}$ \\
\hline $\begin{array}{l}\text { Cannibalization and Asset } \\
\text { Reallocation }\end{array}$ & $\begin{array}{l}\text { - Reduced launch mass for spares } \\
\text { Ability to mitigate unexpected } \\
\text { failure }\end{array}$ & $\begin{array}{l}\text { More complex repairs, e.g. EVA } \\
\text { to retrieve parts } \\
\text { More complex mission } \\
\text { architecture, such as delayed } \\
\text { jettison of expired elements }\end{array}$ \\
\hline
\end{tabular}




\section{A. Level of Repair}

Levels of repair on spacecraft can range from large assemblies, such as Orbital Replacement Units (ORUs), to small internal components. Repair of failed components at lower levels allows for sparing smaller components and focusing on the items most likely to fail. In many components the largest mass and volume are in casings and mounting hardware that are very unlikely to fail. Failures are much more likely to occur in certain internal components such as electronic cards, motors, switches, seals and numerous other small items. However, repairs at the box level can be much simpler to execute and thus to train the flight crew to perform. Lower repair levels also require more specialized tools, workbenches and test equipment to verify successful repairs.

Components must be designed and built to be accessible for in-flight maintenance of components inside the box. Use of common tools to open up and disassemble components will significantly increase the ability to perform lower levels of repair. However, lower level of repair will also require improved diagnostic capabilities. While it is generally obvious which component has failed at the box level, it often more difficult to determine what has failed at lower levels. The crew will have to have the ability to test failed components, determine which elements need to be fixed or replaced, and then have the information required to complete that repair.

The ISS has successfully implemented internal box level repairs in several instances. The ISS MultiplexerDemultiplexers (MDM) are the spacecraft flight computers, some 40 processers distributed around the vehicle both internally and externally mounted. Each MDM contains several cards, and by sparing at the card rather than the box level, ISS has saved significant mass and volume. ISS has a Maintenance Work Area that supports component repairs that includes mounting hardware, enclosures to keep debris and contaminants out, and to assure containment of tools and equipment. These types of repair facilities are a necessity for successful component repairs, assuring that planned repairs can be conducted, but also accounting for unanticipated repairs to the maximum extent.

Component level repairs will in some cases require refined technical skills such as precision soldering and machining. These skills are developed over years by dedicated technicians, and comparable skills will not be developed to the same degree as part of a traditional mission training regimen. ISS experiments with repairs requiring soldering have achieved less than full success, in part because the crews lacked the skills that trained technicians have developed. Astronaut selection for long-duration missions could include these types of technical skills among the astronaut core. An emphasis on precision robotics to conduct highly technical repairs is another alternative.

Another major issue with lower level repair is the additional time requirements that could be placed on the crew. Typically, the lower the level that maintenance and repair actions take place, the greater the time requirement to complete those actions. Lower level repair can require additional time for access, disassembly, diagnostics, reassembly, trouble-shooting, and testing. With the complexity of systems on a DSV, if Supportability is not carefully planned, there is potential to overwhelm the crew with required maintenance and repair actions.

\section{B. Commonality}

Commonality holds promise for reducing spares mass and volume and reducing cost for development and procurement. Yet its high promise has been notoriously difficult to achieve. Commonality of components, materials and tools would contribute significantly to Supportability by increasing the number of failures that are mitigated for a given type of spare. Interchangeable components, whether or not they are identical, also allow for swapping between elements without necessarily using a spare component. For example, a failing component on a critical module could be replaced by an identical component from a non-critical module.

Successful commonality strategies require an early and enduring programmatic commitment. Most spacecraft have common functions that are developed with similar designs. Avionics, power, thermal, Environmental Control and Life Support System (ECLSS) and other subsystems used in human space programs afford considerable opportunity for use of common components. While there may be significant differences in environments, component lifetime, and performance needs, it is possible to identify similar classes that are candidates for commonality.

In spite of similarity of function, some of the reasons that commonality is not in wider use in spaceflight programs include:

- Programs and projects need to optimize the design for their specific environment and performance requirements, and components in common with other spacecraft may not meet, or may exceed, their needs.

- Spacecraft managers need to be in control of their own specifications. Using a component in common with another spacecraft where the other program/project controls the specification could result in design changes that would no longer meet the needs of the spacecraft that is dependent on the other spacecraft's component.

- Components available for use by a later developed spacecraft may be heading for obsolescence, and the later program needs to take advantage of the latest available mature technology. Avionics developed in the early 2010s may not be a good candidate for a spacecraft that will have a Preliminary Design Review in 2020. 
To address these impediments to commonality, it must be built into the architecture from the earliest program formulation decisions. Very early in program formulation, organizational studies to determine the most effective methods for enabling subsystem commonality across the spacecraft should consider the use of subsystem projects and contractors, or use of directed subcontracts. Commonality of avionics, power systems, thermal, ECLSS, propulsion and other major subsystems can be enhanced by procuring subsystems from a single source, providing the subsystems as government furnished equipment to the vehicle element organizations.

The Constellation Program conducted a Common Avionics Study in spring of 2007, after the program System Requirements Review was completed and projects were working toward their System Design Review. The study concluded that while technical solutions existed, the implications to the projects necessary to realize commonality would be profound. Had the organizations been originally established to achieve commonality, there was a very substantial potential for cost savings in development and operations. The ISS program achieved commonality of many avionics and power system components by procuring from a single organization that provided the components to other development groups.

\section{Repair During Assembly and Checkout}

Launch of the Supportability material - tools, spares, and materials - requires careful planning and flexibility. Launching spares late in a multi-launch mission allows for adapting spares manifests to known failures or anomalies prior to departure from Earth staging points. Spares to be taken along beyond Earth orbit are needed for Loss of Crew (LOC) and Loss of Mission (LOM) mitigation where there are no possibilities for rapid abort or Earth supported replenishment. Since using spares before departing Earth staging could jeopardize crew safety and mission success beyond Earth orbit, a logistics approach that assures a capability for launch on demand prior to Earth departure is highly advantageous.

Two possible approaches will assure that failures occurring during the Earth environment assembly sequence can be repaired without depleting mission sparing for the critical solar system phases of the mission. The first is to maintain considerable margin for spares on the later launches. Parts, tools and equipment needed to repair any failures discovered during transit to staging areas or in uncrewed assembly at the staging point should be able to be manifested, with very short notice, on any of the remaining launches after discovery of the failure. The second approach is to use a separate uncrewed logistics launch. A separate launch could facilitate replacement of large element failures, such as an engine or a complete spacecraft module. Maintaining ample launch schedule margin, as well as destination flexibility, will help prevent a significant module degradation or loss at the Earth staging assembly orbit from causing a total loss of mission.

\section{Redundancy}

Redundancy strategies for multi-element solar system exploration spacecraft afford opportunities for new approaches that could significantly improve Supportability, as well as several challenges.

Sparing and redundancy are heavily intertwined. Where commonality is used, a single spare mitigates failures in multiple locations, and if rapid failover time is not required, stored spares provide advantages over built-in redundancy. For unique components, there may be little difference between storing a spare in inventory, and adding an additional level of built-in redundancy. A stored spare could simplify the design to support failover to another component, but require additional procedures, training and tools needed to perform the in-flight maintenance.

Functional redundancy across spacecraft modules can reduce the need for maximum redundancy in each module. If avionics, power, ECLSS, thermal and other functionality from one module can be used to mitigate loss of those functions in another module, the criticality of each module is reduced. Spacecraft level reliability and criticality analyses will be required to achieve an optimum balance between component redundancy within a spacecraft module and functional redundancy. As long as the spacecraft can be assured of providing critical functions from somewhere, it will not be necessary to treat every module's subsystems as high criticality components. Optimizing across the system will reduce the criticality within each module. For example, if a power system failure in any module can be mitigated by power systems in other modules without threatening a loss of mission or crew, each subsystem can be designed with less redundancy. Coupled with commonality, the combined mass of in-use components and spares can be significantly reduced from systems that are designed independently.

\section{E. Reliability}

Reliability figures prominently in determining LOC and LOM. Redundancy can mitigate many failures, although in a multi-month mission with no capacity to resupply and where aborts will still require up to several months to complete, the possibility of multiple failures must be considered, and prospects for lengthy durations when one failure away from criticality must be carefully considered. While it may be tempting to make assumptions that 
technology and time will allow for substantially improved reliability and reduce the need for Supportability and redundancy, such assumptions should be avoided unless strong evidence exists to support the assumption.

In general most components are quite reliable. Spares mass is not driven by systems with poor reliability, but rather by the sheer number of potential failures on complex spacecraft. Most spares will not be used, but they must be carried as risk mitigation. The inclination to adopt a policy of mitigating risk with "we'll just make the systems more reliable” is a policy not supportable by data.

\section{F. In-Space Manufacturing}

Several approaches have been proposed and researched to provide capabilities to manufacture replacement parts. One of the more promising technologies is the Electron Beam Freeform Fabrication $\left(\mathrm{EBF}^{3}\right)^{\mathrm{iii}}$ process that has been developed at NASA Langley Research Center and by others over the past several years. This process is capable of building metallic components from a wire or powder feedstock, using a CAD type pattern to create a quite wide range of alloys and metallic characteristics. Precision parts will generally require some additional machining and polishing to meet final component specifications. Verification of manufacturing will require techniques such as 3-D scanning to determine the exact shape. The $\mathrm{EBF}^{3}$ process is also capable of making repairs to damaged items. The electron beam could first be used to ablate damaged material, and then build up the damaged area to restore the original shape. Additionally, in-space manufacturing capabilities could focus on manufacturing tools. Custom tools are generally required to complete repairs and the ability to fabricate these tools on orbit could reduce mass and volume requirements.

It is far from clear, however, if manufacturing capabilities could significantly change the sparing mass and volume needs for long-duration exploration missions. Based on data provided by the ISS Logistics and Maintenance Office, the ISS conducted over 1500 unscheduled maintenance actions between 1998 and 2010, and few if any could have been accomplished with in-space manufacturing techniques. Many failures involve complex components with electronics, fluid systems, or chemical processing that are not amenable to repairing with parts that can be manufactured by available techniques. Often the specific failure is not known until the failed component is returned to Earth and detailed engineering analyses are conducted.

These techniques have significant merit from a "what if" analysis perspective. No logistics analysis can assure that all contingencies are covered. Something is likely to break, defying all the probabilistic analysis that said it would never happen. Manufacturing capabilities such as $\mathrm{EBF}^{3}$ and other similar techniques could prove crucial to long-duration space missions under some circumstances, such as micro-meteoroid object damage that strikes a critical spacecraft component. These types of techniques should be developed and used for long-duration missions, not necessarily to reduce sparing needs, but rather as a means of giving the crew their best "fighting chance" to survive unforeseen circumstances.

\section{G. Cannibalization and Asset Reallocation}

A planned approach for scavenging parts from expired modules prior to jettison or discard, and prioritization between modules, can contribute to logistics to maximize Supportability with minimum extra mass. Exploration mission architectures may include discarding modules along the way. Spacecraft designs can be developed such that spares stocks can be built up from scavenging parts before jettisoning expired modules. For example, some architectures assume elements are left at the destination; therefore critical common spares (e.g. avionics) could be scavenged from those systems before departure from the destination. Removing key components prior to discard could provide spares for the long-duration phases of the mission without incurring any additional launch mass. Automated or robotic removal and storage would be required for most of these as much of the jettisoning occurs before crews arrive. Avoiding extensive EVA work would make this a more attractive option.

Once the crew arrives and the extended mission phase has begun, some repairs could be conducted by using a less critical component to replace a higher criticality failure. Criticality will vary by module and mission phase. For example, a crew re-entry vehicle backup component could be removed and used in a detachable surface exploration vehicle at the exploration destination to repair a critical failure; once the destination exploration activity is completed, the component could be replaced in the re-entry vehicle to assure the function is available for Earth return. These types of interchange between modules could further reduce the mass and volume of spares without reducing the probability of repair and thus the probability of mission success and safe crew return.

Commonality and interchangeability of components between modules is required in order to realize significant benefits. Reusing and repurposing dissimilar components may occasionally be necessary with creative engineering to solve unexpected problems, but with logistics planning for reuse and repurposing of selected components, the overall spares allocation can be reduced. 


\section{Model and Forward Work}

Current methodologies of determining Supportability requirements utilize historic analogies and estimates of mean time between failures to help quantify the requirements. In addition, parametric sizing sensitivity analyses are employed to determine the effects on the exploration systems in underestimating or overestimating logistics requirements. An example of parametric sizing sensitivity analysis is shown in Figure 3, which highlights the propellant mass and total mass as the logistics mass is varied for an all chemical propulsion near-Earth asteroid mission. The analysis was completed using an in-house parametric system modeling tool called Exploration Architecture Model for IN-space and Earth-to-orbit (EXAMINE) ${ }^{\text {iv }}$. As expected, the propellant mass and total mass increase as the logistics mass is increased; however, this approach does not account for the complexities of the problem, including crew time requirements, the reliability of parts and systems, and the maintenance requirements.

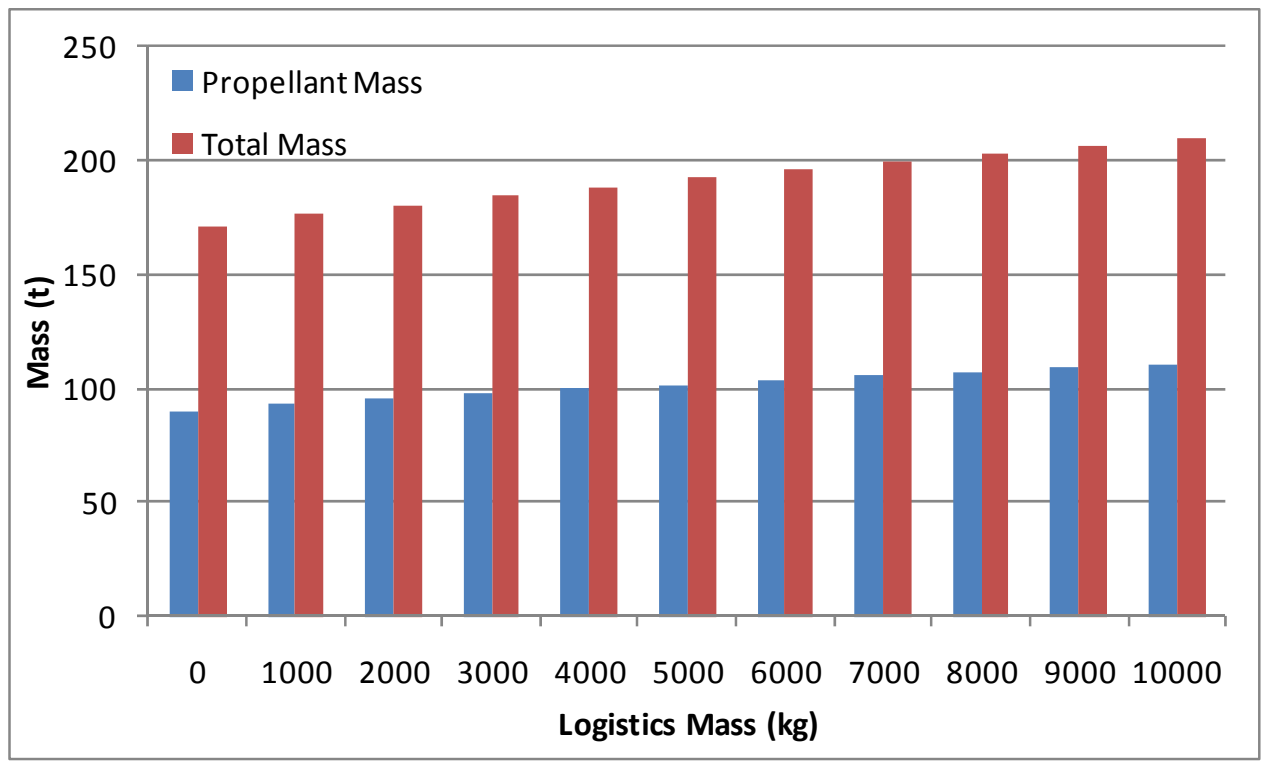

Figure 3 - Parametric Sizing Sensitivity Analysis Example

Logistics resupply mass and volume may drive architectural level designs. As seen in Figure 3, if the appropriate logistics mass is not accounted for during the conceptual design cycle and throughout program formulation, the life cycle costs of the program may be higher than anticipated due to the addition of more launch vehicles required to deliver the required logistics mass. In addition, the amount of required logistics mass may invalidate the design of the DSV if considerable mass or volume is required for Supportability and not initially included.

To obtain a better estimation method for determining the logistics requirements for beyond LEO missions, the authors are in the process of developing a model that dynamically simulates maintenance, failures, and repair actions on key systems in the elements that make up a DSV. The model will not simulate the operations of the systems themselves, but rather track the actions and materials required to maintain and repair the systems and evaluate the overall reliability of the systems based on the Supportability.

The basis of the Supportability simulation is a basic format for describing the makeup of each system. This description will not detail the operations of the system but rather describe the components that make up the system and their criticality to overall system operation, including redundancy. Descriptions will be made using a standardized taxonomy that breaks systems down into components and sub-components. The taxonomy will be designed to match the possible levels of repair and maintenance. It is anticipated the system descriptions will be general in nature, in that the actual system designs are not available. The description will focus on the functionality of each component and sub-component and on the type of processes employed.

Along with the system description will be a matching reliability database. This database will be based on the same structure as the system description and will contain data on the reliability of each component and subcomponent. Reliability data will be developed based on heritage data from similar systems or from subject matter expertise (SME) input for developmental systems.

A matching maintenance and repair database will also be developed. This database will initially contain a mass estimate for prototypical maintenance and repairs for each component and sub-component. The database will contain estimates for different levels of repair-for example, mass estimates will be made for repairs to failures that 
occur at the component level, the sub-component level, and the board level. Maintenance estimates will be timebased and capture the mass required for preventive maintenance for each system and sub-system. Because the actual systems are not designed, all estimates for maintenance and repair masses will be 'average best estimates', based on heritage data and analysis of future system operation. The masses are not intended to represent specific maintenance and repair items, but rather are intended to represent generic items. In future iterations, this database could also include average required crew hours for maintenance and repair at different repair levels.

The final database is a maintenance and repair item inventory. This database captures all of the maintenance and repair items that are manifested on a deep space mission. The format of the inventory is keyed to the maintenance and repair database described above. The inventory lists the quantity of each generic repair item, including a specification of the intended level of repair. The database will also include an option for specifying commonality and scavenging between repair items. This indicator will specify where the repair items between two or more components or sub-components are common.

The actual Supportability model is a Monte Carlo simulation that uses the described databases to simulate system Supportability during a deep space mission. Using the system description and reliability database, the model simulates potential failures during a specific mission run. The model then checks the inventory to see if the specific failure can be repaired, subtracting from the inventory if it can. If the failure cannot be repaired, the model uses the criticality description to evaluate the resultant mission impact. In addition, the model tracks required maintenance mass for all systems. Figure 4 gives a visual representation of the model.

The model will be used to estimate the total maintenance and repair mass required to achieve different levels of overall mission reliability. Scenarios can be evaluated with different maintenance and sparing strategies, including level of repair, repair item inventory, commonality and scavenging, and level of redundancy. The model will also be used to investigate the impact of increased system reliability on sparing. If crew maintenance and repair times are included, the model could then be used to balance level of repair with crew time constraints.

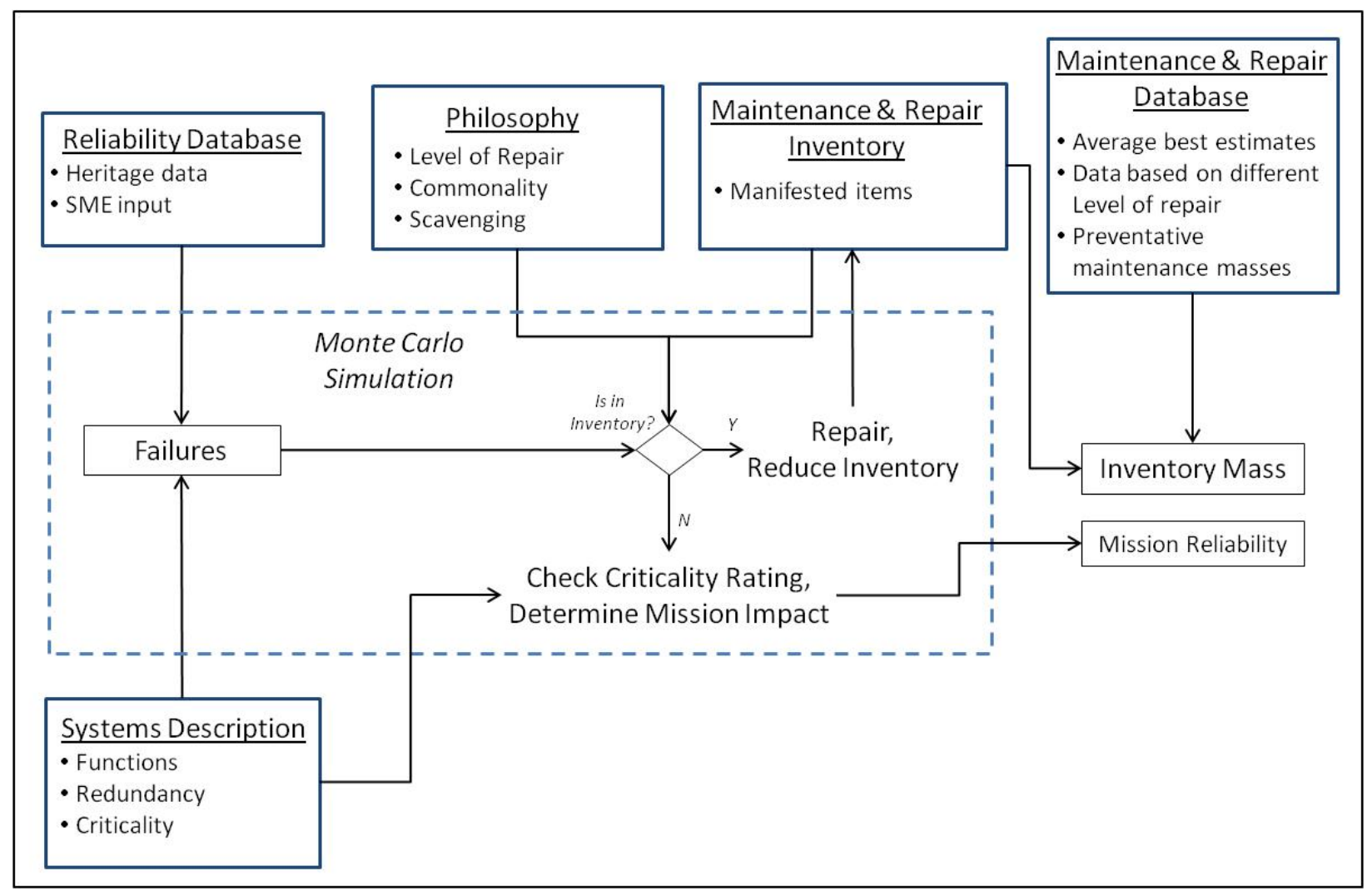

Figure 4 - Supportability Model Flow Chart 


\section{Summary}

As NASA continues to investigate options to explore beyond low Earth orbit, various approaches for Supportability of these missions will be analyzed. Supportability strategies will impact deep space mission hardware and operations through probability of system failure, mission risk, systems accessibility, crew time requirements to maintain and repair systems, and the amount of spares and maintenance items required for missions. Various methods for improving Supportability include level of repair, commonality, assembly repair, redundancy, reliability, in-space manufacturing, and cannibalization and asset reallocation. To understand the impacts of Supportability strategies, the authors are in the process of developing a model to dynamically simulate maintenance, failures and repair actions on key systems typical of a deep space vehicle, track the actions and materials required to maintain and repair the systems and evaluate the overall reliability of the systems.

\section{Acknowledgments}

The authors would like to acknowledge Hilary Shyface of Analytical Mechanics Associates, Inc. for the logistics parametric sizing sensitivity analysis she provided. Additionally, the authors would like to acknowledge the ISS Logistics \& Maintenance Office at Johnson Space Center for providing ISS data and sharing their acquired knowledge.

\section{References}

\footnotetext{
i NASA Systems Engineering Handbook, National Aeronautics and Space Administration, NASA/SP-2007-6105 Rev1, Washington, D.C., December 2007, pp 65-66.

${ }^{\text {ii }}$ Culbert, C., Martin, E., Mongrard, O., Satoh, N., Goodliff, K., and Troutman, P., "ISECG Mission Scenarios and their role in informing Next Steps for Human Exploration beyond LEO,” $62^{\text {nd }}$ International Astronautical Congress, Cape Town, South Africa, 2011.

iii Hafley, R. A., Taminger, K. M. B., and Bird, R. K., "Electron Beam Freeform Fabrication in the Space Environment," 45 AIAA Aerospace Sciences Meeting and Exhibit, AIAA 2007-1154, Reno, NV, 2007.

iv Komar, D. R., Hoffman, J. A., Olds, A. D., and Seal, M. D., "Framework for the Parametric System Modeling of Space Exploration Architectures,” AIAA Space 2008 Conference and Exposition, AIAA 2008-7845, San Diego, CA, 2008.
} 